Western University

Scholarship@Western

Physical Therapy Publications

Physical Therapy School

5-1-2017

\title{
The Attentional Demands of Ambulating with an Assistive Device in Older Adults with Alzheimer's Disease
}

Susan W. Hunter

Western University, smuir4@uwo.ca

M Montero-Odasso

Follow this and additional works at: https://ir.lib.uwo.ca/ptpub

Part of the Physical Therapy Commons

Citation of this paper:

Hunter, Susan W. and Montero-Odasso, M, "The Attentional Demands of Ambulating with an Assistive Device in Older Adults with Alzheimer's Disease" (2017). Physical Therapy Publications. 31.

https://ir.lib.uwo.ca/ptpub/31 
The attentional demands of ambulating with an assistive device in older adults with Alzheimer's disease

SW Muir-Hunter ${ }^{1,2}$, M Montero-Odasso $^{2,3}$

1. School of Physical Therapy, University of Western Ontario, London, ON, Canada. 2. Department of Geriatric Medicine, Schulich School of Medicine \& Dentistry, University of Western Ontario, London, ON, Canada. 3. Gait and Brain Lab, Parkwood Hospital, London, Ontario, Canada.

Corresponding author:

Dr. Susan Hunter

University of Western Ontario

School of Physical Therapy

Room 1588, Elborn College

London, Ontario, Canada N6G 1H1

Phone: 519-661-2111 ext 88845

Email: susan.hunter@uwo.ca

Acknowledgements: The authors thank Karen Johnson, Director of Alzheimer Outreach Services of McCormick Home; Steve Crawford, CEO, WCA; and the staff and clients at the Alzheimer Outreach Services day program for their hospitality, assistance in organizing this project and participation in the data collection process. This study was funded by the Physiotherapy Foundation of Canada Seniors' Health Division Research Award in Older Adult Health and had no involvement in the conduct of the study.

Keywords: aged, gait, assistive devices, walkers, dementia 


\title{
Ambulation with a walker during complex path configurations induces a higher attentional cost for persons with Alzheimer's disease
}

\begin{abstract}
Ambulation with a mobility aid is a unique real-life situation of multi-tasking. These simultaneous motor tasks place increased demands on executive function in healthy young and older adults, but the demands have not been evaluated in people with Alzheimer's disease (AD). Mobility problems are common among adults with $\mathrm{AD}$, leading to provision of a mobility aid to optimize independent activity. The study objectives were: i) to determine the dual-task cost (DTC) associated with the use of a mobility aid in straight and complex path walking, and ii) to evaluate the association between executive function and ambulation with a mobility aid in older adults with $\mathrm{AD}$ and age-sex matched cognitively normal controls. Fourteen people (mean age \pm SD, $72.6 \pm 9.9$ years) with a diagnosis of probable AD (MMSE range 12-25) and controls (mean age $\pm S D, 72.9 \pm 9.5$ ) walked at a self-selected pace and using a 4-wheeled walker in a 6 meter straight path and a Figure of 8 Test. Ambulation with the walker in a straight path produced a low DTC that was not different between the groups. Ambulation with the 4-wheeled walker in the complex path produced a significantly different DTC in the group with AD at $38.1 \pm 23.5 \%$ compared to $-19.7 \pm 21.4 \%$ ( $\mathrm{p}=0.041$ ). Lower scores on executive function were associated with longer times across test conditions. Ambulation with a 4-wheeled walker, in particular maneuvering around obstacles, requires greater attentional costs in dementia. Future research should explore the timing for safely introducing mobility aids in $\mathrm{AD}$ and the role of improving executive function.
\end{abstract}




\section{INTRODUCTION}

Falls among older adults are a significant public health problem and have substantial consequences on an individual's quality of life and independence. An emerging area of falls research is the role of cognition in postural stability; a timely focus as the prevalence of dementia, and its attendant increased health care needs and utilization, is expected to increase dramatically in coming years.[1]

Ambulation with a mobility aid is a unique real-life situation of multi-tasking; the performance of these simultaneous motor tasks places increased demands on brain resources related to executive function.[2] Executive function, which comprises the set of cognitive processes that use sensory information to modulate behavior, is required for planning movements, dividing attention, and responding to changes in the environment.[3] Increased attentional demands have been observed in healthy young adults [4] and older adults[5] using mobility aids suggesting that executive resources are necessary for their proper use. The use of a mobility aid requires high levels of motor control and may challenge cognitive function to generate responsive motor patterns to multiple sensory inputs and environmental conditions.[2]

Decreased executive function is an early symptom in Alzheimer's disease (AD)[6] and a proven risk factor for falls[7]. In addition, gait and mobility impairments are common features in community-dwelling older adults living with dementia.[8] Older adults with cognitive impairment demonstrate decreased gait velocity and increased gait instability while multitasking, which are also associated with an elevated fall risk.[9] Rehabilitation interventions to reduce risk can include individualized exercise programs and the prescription of a mobility aid to compensate for deficits and allow maintenance of independent ambulation.

The use of a mobility aid is also associated with an elevated fall risk in older adults.[10] Mobility aid use could be a proxy for the presence of intrinsic functional limitations, such as balance or gait problems; but, the elevated risk remains even when those factors have been taken into account.[10] Additional considerations that would link mobility aid use to an elevated fall risk include interference with lower extremity movement during balance recovery to a perturbation, it prevents the use a person's hands to effectively reach for support when there is a loss of balance and it increases cognitive demands related to attentional processing and neuromotor control.[2]

Observing people during a gait or balance task while they perform a secondary task (the dual-task paradigm) is an accepted way to assess the interaction between cognition and mobility.[11] Cognitive demands relative to the cognitive capacity or reserve of the individual influence physical task performance. If the demands of executing two tasks simultaneously exceed the cognitive capacity, then overall performance will be degraded.[12] The configuration of the walking pattern in the dual task testing protocol is also relevant in the evaluation of functional abilities. Gait performance under a straight path condition is considered a low challenge activity, while curved or complex path walking can provide meaningful information about daily life walking ability, including adaptation of walking patterns to negotiate obstacles, change directions, or plan a path.[13]

There has been no research evaluating the "cost" on gait performance of using a mobility aid in older adults with dementia. Moreover, whether the use of assistive devices generates a "cognitive cost" on gait performance in older adults with AD is unknown though it is hypothesized that ambulation with a mobility aid for people with dementia will be associated with a high attentional load. We hypothesized the use of a mobility aid in older adults with AD 
will adversely affect gait performance and be most affected under the test condition of walking with a mobility aid on a curved path test condition. The study objectives were: i) to determine the dual-task cost associated with the use of a mobility aid in straight path and complex path walking in older adults with Alzheimer disease and age-sex matched cognitively normal controls, and ii) to evaluate the association between executive function and the functional performance on ambulation with a mobility aid.

\section{METHODS}

$\underline{\text { Study subjects }}$

A convenience sample of adults with a diagnosis of mild to moderate Alzheimer's disease was recruited from a day program for community-dwelling older adults with dementia. Referral to the day program is based on a confirmed diagnosis of dementia by a geriatrician according to the criteria of the National Institute of Neurologic and Communicative Disorders and Stroke-AD and Related Disorders Association (NINCDS-ARDRA).[14] Inclusion criteria: age greater than 50 years, medically stable, English speaking, no physical impairments that would necessitate use of a mobility aid, had not used a walker previously and able to understand simple instructions. People were excluded if they had any neurological, musculoskeletal, or cardiorespiratory impairment that could compromise safe administration of the testing protocol. All participants or their caregivers provided written informed consent prior to participation in the study.

Control participants were recruited by newspaper advertisement and from a community fitness program. The inclusion criteria for the control group were: no subjective memory complaints, normal performance on cognitive tests, absence of functional impairment, and ability to walk independently. The control participants were matched to the participants with dementia on sex and age, plus or minus 2 years. This study was approved by the Research Ethics Board for Health Sciences Research Involving Human Subjects.

Medical and Cognitive Assessments

Sociodemographic information, co-morbidities, physical activity level, activities of daily living (instrumental and basic), Falls Efficacy Scale-International[15] and medications were recorded. Cognition was assessed using the Mini-Mental State Examination[16] (MMSE; score 0-30). The severity of dementia was categorized according to the MMSE score: mild dementia, MMSE > 20 points; moderate dementia, MMSE = 10-20 points; and severe dementia, MMSE < 10 points. Executive function was measured using the Trail Making Test[17] (TMT). The TMT has two parts: Part A (TMT-A) requires participants to draw lines sequentially connecting 25 numbers, and Part B (TMT-B) requires them to draw lines sequentially alternating between numbers and letters (e.g., 1, A, 2, B, . . ). The TMT assesses visual search ability, scanning, speed of processing, mental flexibility, and executive functioning.[18] Results are reported as the time in seconds required to complete the task; a longer completion time indicates greater impairment. The TMT is sensitive to a variety of neurological impairments and processes.[18] Gait Assessment

In the straight path walking, participants were timed while walking at a self-selected usual speed for a 6 meter distance, with one meter at either end to allow for acceleration and deceleration. In the complex path walking, the Figure 8 Walk Test[19] protocol will be used and reliability for this test has been demonstrated in older adults with dementia.[20] Participants start in standing, midway between two cones placed 1.52 meters apart and walk in a figure-of- 8 path around the cones. The total time and the number of steps was recorded. The dual-task test 
condition required the person to perform both walking configurations using a four wheeled walker.

Sample size. Based on previous data from our research in people with dementia (dual-task cost: $15 \%$ in cognitively normal older adults, $38 \%$ in older adults with dementia)[9], a sample size of 12 participants was needed for a power of $80 \%$ with $\alpha$ error of $5 \%$ to detect a $15 \%$ difference in dual-task cost.

Data analysis: Baseline characteristics of demographic and mobility variables were calculated as means and SDs or frequencies and percentages, as appropriate. For the first objective, a two-way repeated measures ANOVA using a general linear model evaluated the time to complete each walk in each configuration and the number of steps in the complex path ( 3 models). When interactions were non-significant main effects were assessed. The dual-task cost percentage for time to complete the straight path and complex path, and the number of steps in the complex path were evaluated with unpaired t-tests between the controls and the people with AD. The dual-task cost (DTC), as a percentage, was calculated as [(single task value - dual task value) / single task value $] \times 100 \%$ for each walking condition, straight and curved path walking, under single and walking with the wheeled walker and the number of steps to complete the complex path with a walker for each group. A negative DTC indicates that gait performance was decreased under the condition of using the wheeled walker. For the secondary objective, linear regression analyses between executive function (Trail Making Test B) and the time to complete each walking test in each test configuration were performed (4 separate models). All statistical analyses were performed using SPSS version 23.0 (SPSS, Inc., Chicago, IL) with statistical significance set at $\mathrm{p}$ $<0.05$.

\section{RESULTS}

Fourteen people with mild to moderate Alzheimer's disease and controls were recruited for the study. Analysis of demographic characteristics demonstrated differences between the two groups on cognitive function and physical activity (Table 1). The differences between groups on cognition were expected as this defines membership in each group. Also there was a difference in the performance of instrumental and basic activities of daily living, the controls demonstrating no deficits and the people with AD possessing deficits in both domains, greater in instrumental activities consistent with the diagnosis of dementia. Physical activity levels also differed significantly between the groups, though a fear of falling and a history of falls were not different.

In the straight path condition, only the main effect of group was significant. People with $\mathrm{AD}$ walked slower than the controls in the single task $(\mathrm{p}=0.021)$ and using a wheeled walker $(\mathrm{p}=0.020)$. In the complex path condition, the interaction of group $\mathrm{x}$ task was statistically significant for time $(\mathrm{p}<0.001)$ and number of steps $(\mathrm{p}<0.001)$. (Figure 1 and 2$)$

The DTC was not different between the controls and people with AD for the wheeled walker task $(-0.53 \pm 10.14 \%$ versus $-4.64 \pm 11.47 \%, \mathrm{p}=0.324)$ under the straight path condition. In the complex path condition, the DTC was significantly different between people with AD and controls for the wheeled walker task $(-38.1 \pm 23.5 \%$ versus $-19.7 \pm 21.4 \%, p=0.041)$ indicating greater cognitive load in the people with Alzheimer's disease. The dual-task cost for the number of steps was also significant $(-16.85 \pm 17.91 \%$ versus $-34.20 \pm 19.61 \%, \mathrm{p}=0.022)$.

The linear regression demonstrated that executive function was significantly related to the time to complete all walking tasks; longer times to complete the TMT-B were associated with longer times to complete the walking tests. (Figure 3) The unstandardized regression coefficients for the TMT-B were: simple path usual gait, $\beta=0.006(0.003-0.01), p=0.001$, 
$\mathrm{R}^{2}=0.373$; simple path with walker, $\beta=0.008(0.003-0.013), \mathrm{p}=0.002, \mathrm{R}^{2}=0.352$; complex path usual gait, $\beta=0.019(0.007-0.032), \mathrm{p}=0.001, \mathrm{R}^{2}=0.291$; and complex path with walker, $\beta=0.024(0.010-0.037), p=0.001, R^{2}=0.346$. The regression coefficient represents the amount of change in time, in seconds, to complete the walking test that is associated with each second increase in time to complete the TMT-B.

\section{DISCUSSION}

This study has demonstrated that ambulating with a walker in a complex pattern generates a significantly higher cognitive load in people with $\mathrm{AD}$, as demonstrated by a larger dual-task cost compared to healthy controls. Our finding is consistent with previous research that has demonstrated an increased cognitive load with use of a walker in healthy younger and older adults[2,4,5]; and, to the best of our knowledge, this study is one of the first to show that people with dementia experience an even greater demand on already limited or fragile cognitive resources. This finding has important clinical implications for fall prevention strategies and mobility aid prescription among people with AD.

Interestingly, the time to complete the straight path with the wheeled walker was no different than the single-task activity of usual gait speed for either group. This highlights the importance of the test configuration for dual-task testing to detect deficits as a straight path is considered a low challenge scenario.[13,22] This study supports that even in people with mild to moderate $\mathrm{AD}$ this is a low challenge activity and does not represent real world situations that are encountered while using a wheeled walker, such as maneuvering around obstacles.

Current recommendations for fall prevention guidelines in people with dementia are limited.[23, 24] Unfortunately the strategies that successfully reduce falls in the cognitively normal have not translated successfully to the cognitively impaired,[26] though there is accumulating evidence of benefit in community-dwelling older adults with dementia[27]. Rehabilitation interventions to reduce fall risk can include individualized exercise programs and the prescription of a mobility aid to compensate for deficits to allow maintenance of independent ambulation. Possible explanations for the overall greater fall risk in people with dementia include different underlying mechanisms, the magnitudes of association for risk factors that are shared with cognitively normal older adults is greater and there may be unique risk factors that are not present in cognitively normal adults. In addition to the development of new strategies, the present study emphasizes we must also understand the impact of our current rehabilitation interventions in order to develop programs that meet the unique health care needs of older adults with dementia.

Impairment in executive function is associated with falls[7], and balance and gait problems in older adults with and without a diagnosis of dementia $[13,22,23]$. This study also demonstrated a significant relationship between executive function and walking, such that lower scores on executive function were associated with longer times to complete each walking task. As hypothesized, walking in a straight path without a walker had the lowest magnitude of association to executive function and walking in a complex path with a walker had the highest. Though the association was statistically significant, executive function only explained a small amount of the variation in the time to complete the walk. As all people were new to using a mobility aid, there may be a greater variation that was not explained by executive function in the people with $\mathrm{AD}$ as a result of limited motor learning of a novel task creating anxiety of using something unfamiliar and lower efficiency with execution of the movement. Further research is 
warranted on the effects of training in people new to use of mobility aids and among people experienced in using a mobility aid.

Difficulty maneuvering around obstacles can be a result of altered visuospatial abilities and executive function, which combined with limited memory for retaining teaching of the safe use of the equipment can pose a fall risk problem. $[27,28]$ The people with AD in this study had a higher prevalence of vision problems than the cognitively healthy controls. Vision impairment in depth perception and contrast sensitivity occurs in up to $60 \%$ of people with AD[30], these changes can affect safe navigation around obstacles with a mobility aid and importantly also increase cognitive load and therefore increase fall risk. The role of vision impairment in balance and gait function among people with AD using a mobility aid has not been evaluated and merits research.

It should be emphasized that the people with $\mathrm{AD}$ in this study were naïve to use of a mobility aid and were not in need of using a mobility aid for ambulation. The study was to evaluate the early learning effects of using an assistive device, independent of any functional limitations that would usually necessitate a prescription for a walker. Future research should focus on the timing of mobility aid prescription and the potential for learning effects with repeated exposure to use of the equipment. It has been demonstrated that people with AD possess intact implicit motor-learning capacities and therefore the potential for a reduction in cognitive load while using a mobility aid is a possibility with training.[32]

There are several strengths to this study. Our samples were age and sex-matched to remove these possible confounders as a source of any differences identified between the groups. We were also able to perform several tests of cognitive function to provide a broad overview of abilities between the groups. Additionally, all participants were able to complete the full assessment protocol without loss of study participants. Some limitations to the study include we did not include another manual task challenge for comparison, such as walking while carrying a glass of water as a comparison of magnitude of cognitive load across different tasks. While a sample size calculation was performed using data from other gait studies in people with Alzheimer's disease, the variation in scores was much greater than expected and may impact magnitude of associations found in this study.

\section{CONCLUSIONS}

Ambulation with a wheeled walker, in particular maneuvering the walker around obstacles, creates a greater attentional load in people with dementia than cognitively healthy peers and presents a potential fall risk. In adults with dementia, the timing of gait aid prescription to facilitate independent mobility may coincide with fragile brain function that cannot accommodate greater resource utilization, ultimately leading to instability, unsafe use of the aid and falls. Future research should explore the timing for safely introducing mobility aids among people with $\mathrm{AD}$ and the role of improving executive function.

\section{Conflicts of interest: none}




\section{REFERENCES}

[1] Alzheimer Society of Canada. Rising Tide : The Impact of Dementia on Canadian Society. Executive Summary. Dementia 2010:1-24. doi:9780973352221.

[2] Bateni H, Maki BE. Assistive devices for balance and mobility: Benefits, demands, and adverse consequences. Arch Phys Med Rehabil 2005;86:134-45. doi:10.1016/j.apmr.2004.04.023.

[3] Yogev-Seligmann G, Hausdorff JM, Giladi N. The role of executive function and attention in gait. Mov Disord 2008;23:329-42; quiz 472. doi:10.1002/mds.21720.

[4] Wright DL, Kemp TL. The dual-task methodology and assessing the attentional demands of ambulation with walking devices. Phys Ther 1992;72:306-312-315.

[5] Wellmon R, Pezzillo K, Eichhorn G, Lockhart W, Morris J. Changes in dual-task voice reaction time among elders who use assistive devices. J Geriatr Phys Ther 2006;29:74-80. doi:10.1519/00139143-200608000-00006.

[6] Perry RJ, Hodges JR. Attention and executive deficits in Alzheimer's disease A critical review 1999:383-404.

[7] Muir SW, Gopaul K, Montero Odasso MM. The role of cognitive impairment in fall risk among older adults: a systematic review and meta-analysis. Age Ageing 2012;41:299308. doi:10.1093/ageing/afs012.

[8] Allan LM, Ballard CG, Burn DJ, Kenny RA. Prevalence and Severity of Gait Disorders in Alzheimer's and Non-Alzheimer's Dementias. J Am Geriatr Soc 2005;53:1681-7. doi:10.1111/j.1532-5415.2005.53552.x.

[9] Muir SW, Speechley M, Wells J, Borrie M, Gopaul K, Montero-Odasso M. Gait assessment in mild cognitive impairment and Alzheimer's disease: The effect of dual-task challenges across the cognitive spectrum. Gait Posture 2012;35:96-100. doi:10.1016/j.gaitpost.2011.08.014.

[10] Deandrea S, Lucenteforte E, Bravi FF, Foschi R, La Vecchia C, Negri E. Risk factors for falls in community-dwelling older people: a systematic review and meta-analysis. Epidemiology 2010;21:658-68. doi:10.1097/EDE.0b013e3181e89905.

[11] Montero-Odasso M, Verghese J, Beauchet O, Hausdorff JM. Gait and Cognition: A Complementary Approach to Understanding Brain Function and the Risk of Falling. J Am Geriatr Soc 2012:n/a-n/a. doi:10.1111/j.1532-5415.2012.04209.x.

[12] Snijders AH, van de Warrenburg BP, Giladi N, Bloem BR. Neurological gait disorders in elderly people: clinical approach and classification. 2007;6:63-74.

[13] Lowry KA, Brach JS, Nebes RD, Studenski SA, VanSwearingen JM. Contributions of Cognitive Function to Straight- and Curved-Path Walking in Older Adults. Arch Phys Med Rehabil 2012;93:802-7. doi:10.1016/j.apmr.2011.12.007.

[14] McKhann GM, Knopman DS, Chertkow H, Hyman BT, Jack CR, Kawas CH, et al. The diagnosis of dementia due to Alzheimer's disease: Recommendations from the National 
Institute on Aging-Alzheimer's Association workgroups on diagnostic guidelines for Alzheimer's disease. Alzheimer's Dement 2011;7:263-9. doi:10.1016/j.jalz.2011.03.005.

[15] Hauer K, Yardley L, Beyer N, Kempen G, Dias N, Campbell M, et al. Validation of the falls efficacy scale and falls efficacy scale international in geriatric patients with and without cognitive impairment: Results of self-report and interview-based questionnaires. Gerontology 2010;56:190-9. doi:10.1159/000236027.

[16] Folstein MF, Folstein SE, McHugh PR. Mini-Mental State: A practical method for grading the state of patients for the clinician. J Psychiatr Res 1975;12:189-98. doi:10.1016/0022-3956(75)90026-6.

[17] Weschler D. The Wechsler Adult Intelligence Scale, Fourth Edition. 4th ed. San Antonio: Pearson Assessments; 2008.

[18] Tombaugh TN. Trail Making Test A and B: Normative data stratified by age and education. Arch Clin Neuropsychol 2004;19:203-14. doi:10.1016/S0887-6177(03)000398.

[19] Hess RJ, Brach JS, Piva SR, VanSwearingen JM. Walking skill can be assessed in older adults: validity of the Figure-of-8 Walk Test. Phys Ther 2010;90:89-99.

doi:10.2522/ptj.20080121.

[20] Blankevoort CG, van Heuvelen MJG, Scherder EJ a. Reliability of Six Physical Performance Tests in Older People With Dementia. Phys Ther 2012;93:69-78. doi:10.2522/ptj.20110164.

[21] Frank JS, Patla AE. Balance and mobility challenges in older adults: Implications for preserving community mobility. Am J Prev Med 2003;25:157-63. doi:10.1016/S07493797(03)00179-X.

[22] Muir-Hunter SW, Clark J, McLean S, Pedlow S, Van Hemmen A, Odasso MM, et al. Identifying balance and fall risk in community-dwelling older women: The effect of executive function on postural control. Physiother Canada 2014;66:179-86. doi:10.3138/ptc.2013-16.

[23] Yogev-Seligmann G, Hausdorff JM, Giladi N. The role of executive function and attention in gait. Mov Disord 2008;23:329-42. doi:10.1002/mds.21720.

[24] Summary of the Updated American Geriatrics Society/British Geriatrics Society Clinical Practice Guideline for Prevention of Falls in Older Persons. J Am Geriatr Soc 2011;59:148-57. doi:10.1111/j.1532-5415.2010.03234.x.

[25] Taylor ME, Delbaere K, Close JCT, Lord SR. Managing falls in older patients with cognitive impairment. Aging Health 2012;8:573-88.

doi:http://dx.doi.org/10.2217/ahe.12.68.

[26] Shaw FE. Prevention of falls in older people with dementia. J Neural Transm 2007;114:1259-64. doi:10.1007/s00702-007-0741-5.

[27] Burton E, Cavalheri V, Adams R, Browne CO, Bovery-Spencer P, Fenton AM, et al. Effectiveness of exercise programs to reduce falls in older people with dementia living in 
the community: a systematic review and meta-analysis. Clin Interv Aging 2015;10:42134. doi:10.2147/CIA.S71691.

[28] Dault MC, Frank JS, Allard F. Influence of a visuo-spatial, verbal and central executive working memory task on postural control. Gait Posture 2001;14:110-6. doi:10.1016/S0966-6362(01)00113-8.

[29] Mazaheri M, Roerdink M, Bood RJ, Duysens J, Beek PJ, Peper CLE. Attentional costs of visually guided walking: Effects of age, executive function and stepping-task demands. Gait Posture 2014;40:182-6. doi:10.1016/j.gaitpost.2014.03.183.

[30] Mendola, A. Cronin-Golomb, S. Corkin JG. Mendola_prevalence of vision problems in AD.pdf. Optom Vis Sci 1995;72:155-67.

[31] Wittwer JE, Webster KE, Hill K. Effect of Rhythmic Auditory Cueing on Gait in People With Alzheimer Disease. Arch Phys Med Rehabil 2013;94:718-24. doi:10.1016/j.apmr.2012.11.009.

[32] van Halteren-van Tilborg I, Scherder EJA, Hulstijn W. Motor-skill learning in Alzheimer's disease: a review with an eye to the clinical practice. Neuropsychol Rev 2007; 17:203-12. 
Table 1. Demographic and clinical characteristics of study participants stratified by cognitive status.

\begin{tabular}{|c|c|c|c|}
\hline Variable & $\begin{array}{c}\text { Older Adults } \\
\text { Controls } \\
(n=14) \\
\end{array}$ & $\begin{array}{c}\text { Older Adults with } \\
\text { Dementia } \\
(n=14)\end{array}$ & $\begin{array}{c}\text { Group Comparison* } \\
\text { (p-value) }\end{array}$ \\
\hline Age in years (SD; range) & $72.9(9.5 ; 58-87)$ & $72.6(9.9 ; 57-85)$ & 0.94 \\
\hline Female, n (\%) & $8(57.1 \%)$ & $8(57.1 \%)$ & \\
\hline Years of education (SD; range) & $15.1(2.7 ; 12-21)$ & $13.4(2.6 ; 6-16)$ & 0.10 \\
\hline Number of medications (SD; range) & $1.8(1.9 ; 0-5)$ & $3.0(2.5 ; 0-9)$ & 0.15 \\
\hline \multicolumn{4}{|l|}{ Co-morbidities, n (\%) } \\
\hline Hypertension & $4(28.6 \%)$ & $3(21.4 \%)$ & \\
\hline Diabetes & $1(7.1 \%)$ & $1(7.1 \%)$ & \\
\hline Osteoarthritis & $4(28.6 \%)$ & $4(28.6 \%)$ & \\
\hline Stroke/TIA & $0(0 \%)$ & $3(1 \%)$ & \\
\hline Vision problems & $9(64.3 \%)$ & $12(85.7 \%)$ & \\
\hline Cardiac problems & $4(28.6 \%)$ & $3(21.4 \%)$ & \\
\hline \multicolumn{4}{|l|}{ Activities of Daily Living } \\
\hline Lawton-Brody IADL (SD; range) & $8(0)$ & $2.71(2.27 ; 0-7)$ & $<0.001$ \\
\hline Lawton-Brody BADL (SD; range) & $6(0)$ & $5.43(0.94 ; 3-6)$ & 0.032 \\
\hline
\end{tabular}




\begin{tabular}{|l|l|l|l|}
\hline Falls in the last 12 months, $\mathrm{n}(\%)$ & $1(7.1 \%)$ & $2(14.3 \%)$ & 0.611 \\
Fear of falling, $\mathrm{n}(\%)$ & $2(14.3 \%)$ & $4(28.6 \%)$ & 0.407 \\
Falls Efficacy Scale (SD; range) & $19.5(4.0 ; 16-30)$ & $3(21.4 \%)$ & 0.891 \\
\hline Physical activity level & $13(92.9 \%)$ & $8(57.1 \%)$ & $<0.001$ \\
\hline $\begin{array}{l}\text { Vigorously active, } \mathrm{n}(\%) \\
\text { Soderately active, } \mathrm{n}(\%)\end{array}$ & $1(7.1 \%)$ & $3(21.4 \%)$ & $<0.001$ \\
\hline Cognitive Tests & $28.7(1.6 ; 25-30)$ & $18.6(3.8 ; 12-25)$ & $<0.001$ \\
\hline $\begin{array}{l}\text { MMSE (SD; range) } \\
\text { Trail making test A (seconds)(SD; range) }\end{array}$ & $39.6(10.7 ; 25.3-67.3)$ & $232.8(111.9 ; 110.0-436.0)$ & $<0.001$ \\
\hline
\end{tabular}

*, group comparison using unpaired t-test, $\mathrm{p}<0.05$ set as statistical significance; Vision problems, the total number of the presence of cataracts, cataract surgery, macular degeneration; cardiac problems, the total number of the presence of myocardial infarction, arrhythmia, atrial fibrillation; TIA, Transient Ischemic Attack; IADL, Instrumental Activities of Daily Living Scale; BADL, Basic Activities of Daily Living Scale; MMSE, Mini-Mental State Examination; MoCA, Montreal-Cognitive Assessment. 
Table 2. Time to complete and number of steps for the walking tasks of single task and ambulation with a 4-wheeled walker in a straight and complex path configuration.

\begin{tabular}{|c|c|c|c|}
\hline & & \multicolumn{2}{|c|}{ Group } \\
\hline & Task & Control $(n=14)$ & $\operatorname{AD}(n=14)$ \\
\hline \multicolumn{4}{|l|}{ Straight Path* } \\
\hline \multirow[t]{2}{*}{ Time (sec) } & Single task & $5.09(0.76)$ & $6.05(1.26)$ \\
\hline & Wheeled walker & $5.11(0.95)$ & $6.34(1.58)$ \\
\hline \multicolumn{4}{|l|}{ Complex Path $\dagger$} \\
\hline \multirow[t]{2}{*}{ Time (sec) } & Single task & $8.28(2.45)$ & $11.25(4.87)$ \\
\hline & Wheeled walker & $9.58(1.81)$ & $15.01(5.60)$ \\
\hline \multirow[t]{2}{*}{ Number of steps } & Single task & $14.86(2.28)$ & $17.36(5.85)$ \\
\hline & Wheeled walker & 17.07 (1.77) & $22.93(7.25)$ \\
\hline
\end{tabular}

Values are means with S.D. in parentheses. *, main effect of group only was significant at $\mathrm{p}<0.05$ in ANOVA; $\uparrow$, interaction (group $\mathrm{x}$ task) was significant at $\mathrm{p}<0.05$ in ANOVA for time and number of steps. AD, Alzheimer disease group. 
Table 3. Dual-task cost of ambulation with a 4-wheeled walker in a straight and complex path configuration.

\begin{tabular}{|l|l|l|}
\hline \multirow{2}{*}{ Straight path time } & \multicolumn{2}{|c|}{ Dual-Task Cost (\%) } \\
\cline { 2 - 3 } & \multicolumn{1}{|c|}{ Control (n=14) } & \multicolumn{1}{|c|}{ AD (n=14) } \\
\hline Complex path time & $-0.53(10.14)$ & $-4.64(11.47)$ \\
\hline Complex path number of steps & $-19.7(21.4)$ & $-38.1(23.5)$ \\
\hline
\end{tabular}

Values are means with S.D. in parentheses. Analyses were significant at $\mathrm{p}<0.05$ for all between group comparisons. Negative values indicate a slower time and an increase in the number of steps while using the 4-wheeled walker. 
Figure 1. The effect of single task walking and walking with a 4-wheeled walker on time to complete a complex walking configuration in controls and people with Alzheimer's disease (AD).

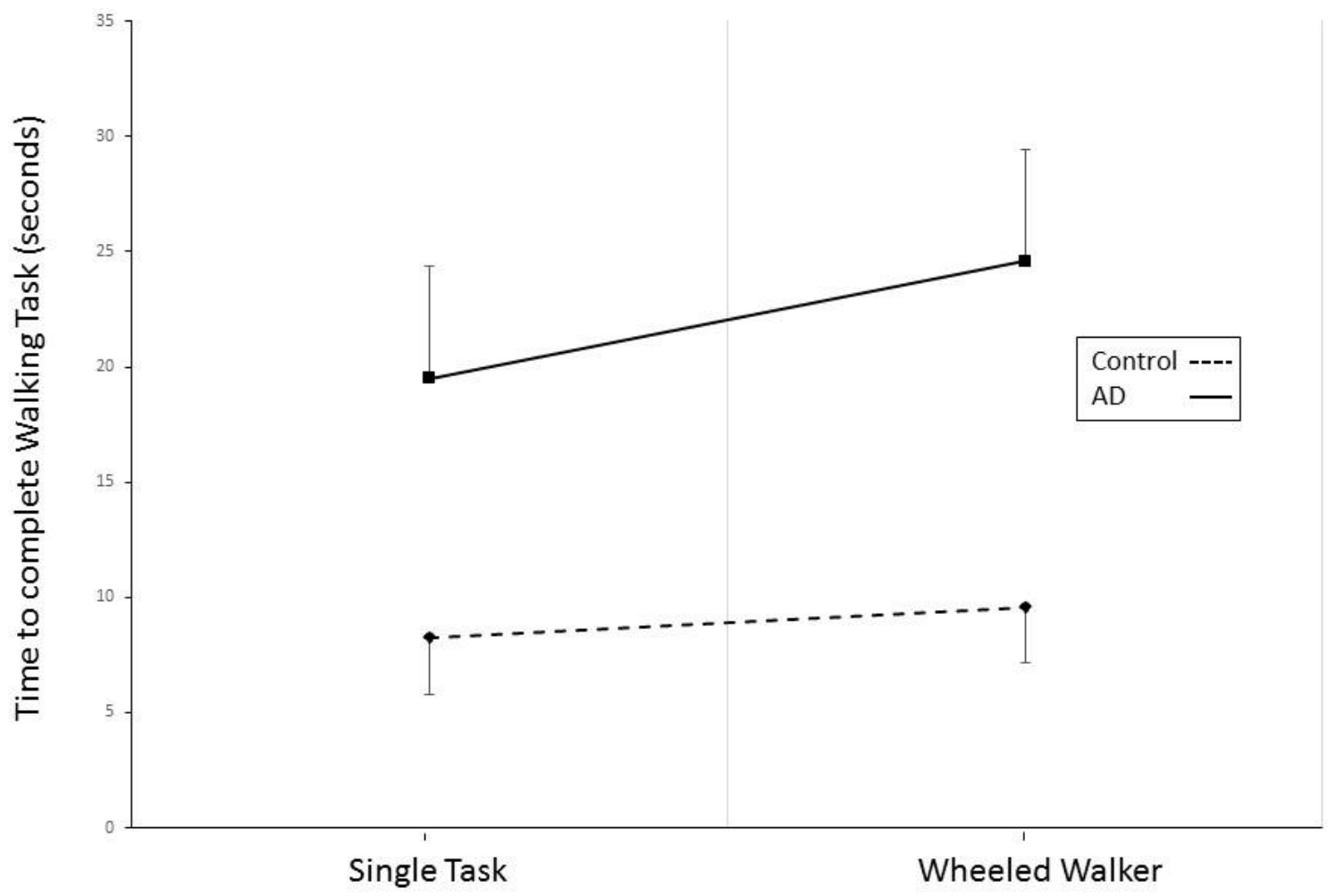


Figure 2. The effect of single task walking and walking with a 4-wheeled walker on the number of steps to complete a complex walking configuration in controls and people with Alzheimer's disease (AD).

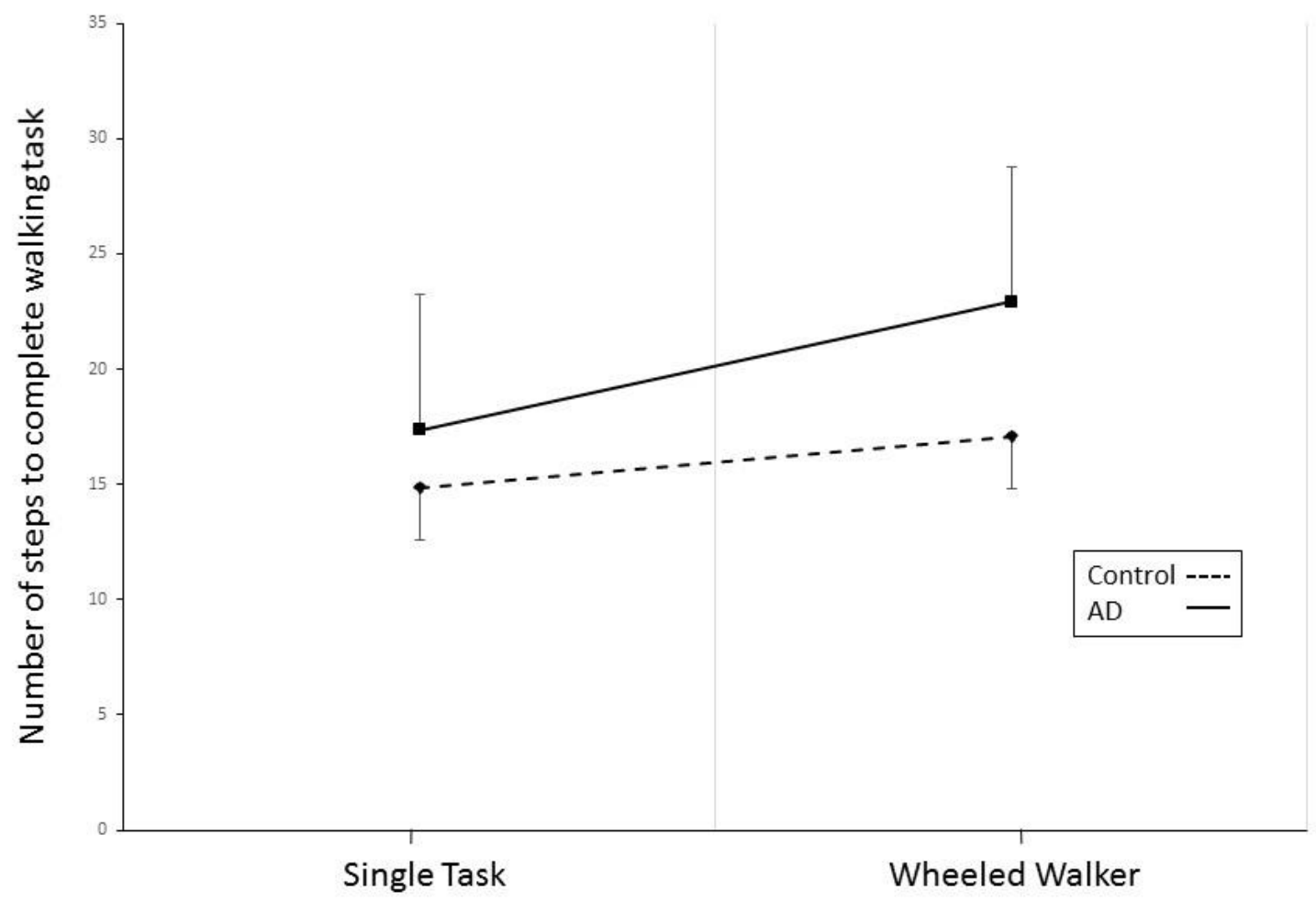


Figure 3. Results of linear regression of association between the time to complete the Trail Making Test-B (TMT-B) and the time to complete the gait tests as a single task and using a 4wheeled walker in a simple path (A) and complex path (B) configuration. 
A.

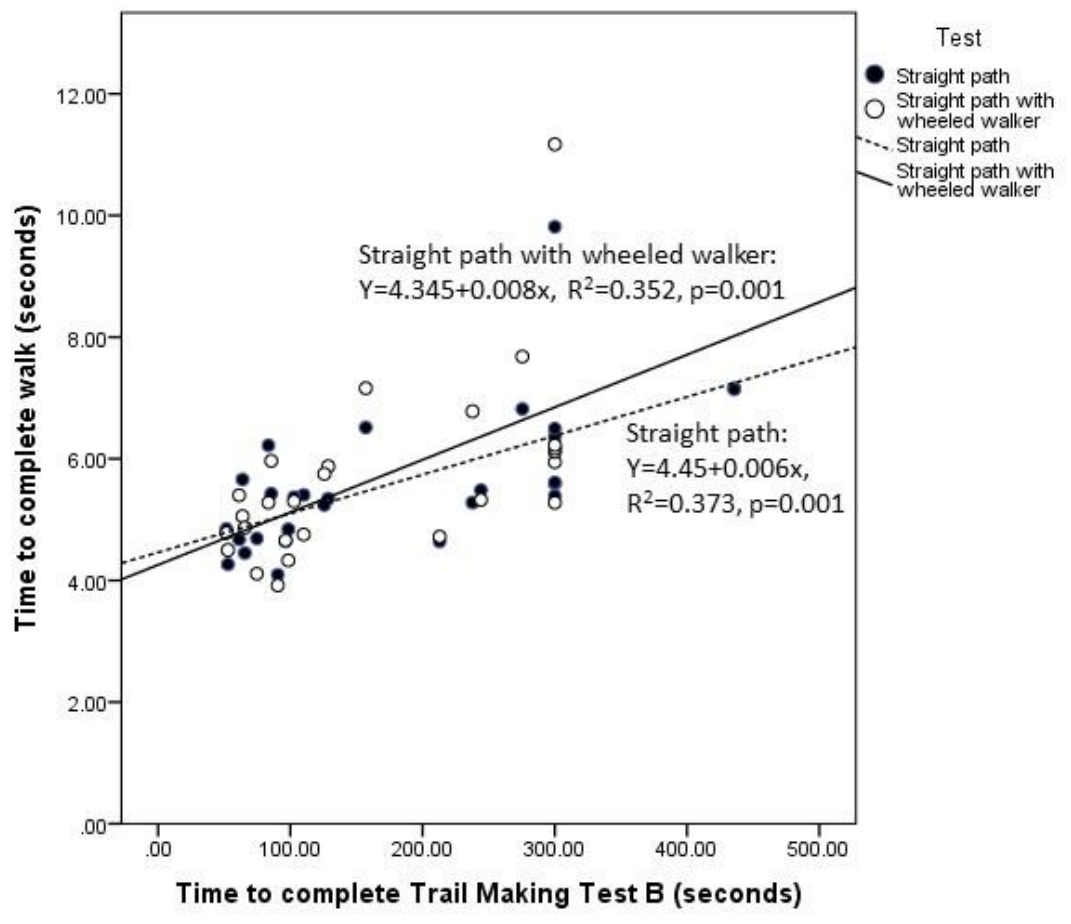


B.

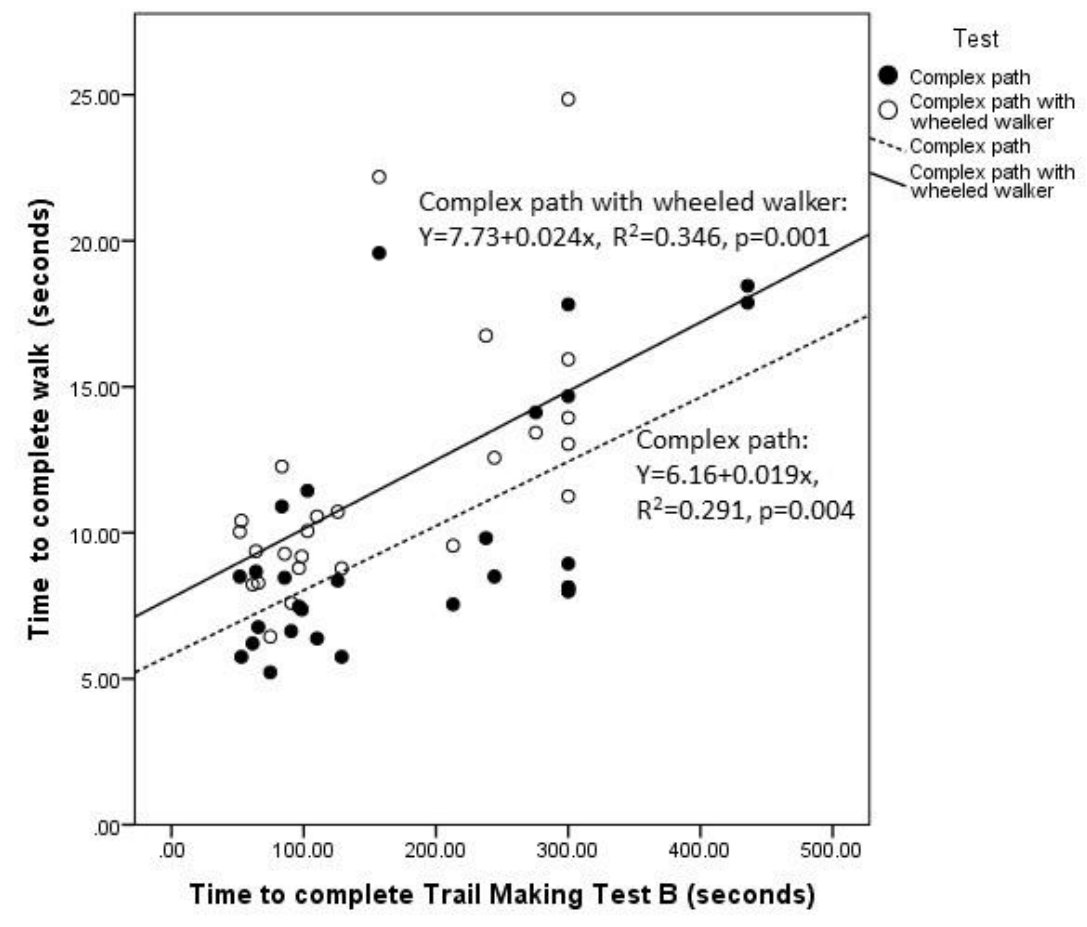

